\title{
REGULARITY FOR OPERATOR ALGEBRAS ON A HILBERT SPACE
}

\author{
JOHN FROELICH
}

(Communicated by Palle E. T. Jorgensen)

\begin{abstract}
Four notions of regularity for operator algebras are introduced. An algebra $A$ is called 1-regular if for any two linearly independent vectors $x, y \in H$ there is an $a \in A$ such that $a x=0$ and $a y \neq 0$. We show that the only weakly closed transitive 1-regular algebra is $B(H)$, thus providing a natural generalization of the Rickart-Yood density theorem. We construct an example of a 1-regular algebra which contains no nonzero compact operators. This example is related to the "thin set" phenomena of classical harmonic analysis.
\end{abstract}

Let $A$ be a commutative semisimple Banach algebra with maximal ideal space $\Gamma$. The algebra $A$ is called regular if for any closed $K \subset \Gamma$ and any $x \notin K$ there is $a \in A$ such that $\hat{a}(K)=\{0\}$ and $\hat{a}(x)=1$. Regular algebras occupy an important position in the commutative theory as they provide an appropriate context in which to study the relationships between elements of the algebra and their Gelfand transforms and especially general spectral synthesis questions. Operator algebras themselves have frequently and fruitfully been viewed as spaces of "noncommutative" functions, for example, the $C^{*}$-algebras as noncommutative topology [2] and reflexive operator algebras as noncommutative harmonic analysis $[1,5,8]$. It seems likely therefore that appropriate notions of regularity can provide useful frameworks for the analysis of operator algebras.

In this paper we introduce four notions of regularity and study their logical relations. We prove that the only transitive 1-regular algebra is $B(H)$, thus providing a natural generalization of the Rickart-Yood transitivity theorem and establishing a surprising connection between these notions and the transitive algebra problem [9]. These notions should also prove useful in studying the interplay between the algebraic structure of an operator algebra and its spatial action.

Throughout the paper we only consider strongly (= weakly) closed algebras of operators on a separable complex Hilbert space $H$. An interesting feature of many of the proofs is the decisive role of the Hilbert space geometry.

Received by the editors April 11, 1992.

1991 Mathematics Subject Classification. Primary 47D25, 47A15. 
Definition 1. An operator algebra $A$ is called:

(i) regular if for any proper subspace $K$ of $H$ and any $y \in K^{\perp}$ there is an $a \in A$ such that $a K=\{0\}$ and $a y \neq 0$;

(ii) IC-regular if for any proper subspace $K$ of $H$ of infinite codimension and any $y \in K^{\perp}$ there is an $a \in A$ such that $a K=\{0\}$ and $a y \neq 0$;

(iii) $F$-regular if for any linearly independent vectors $x_{1}, x_{2}, \ldots, x_{n}$ in $H$ there is an $a \in A$ such that $a x_{1}=a x_{2}=\cdots=a x_{n-1}=0$ and $a x_{n} \neq 0$;

(iv) 1-regular if for any pair of linearly independent vectors $x_{1}, x_{2}$ there is an $a \in A$ such that $a x_{1}=0$ and $a x_{2} \neq 0$.

Clearly (i) $\rightarrow$ (ii) $\rightarrow$ (iii) $\rightarrow$ (iv). We will eventually show that (i), (ii) and (iii), (iv) are equivalent. We mention that $B(H)$, the algebra of all bounded operators on $H$, is an example of a regular algebra.

Proposition 2. (i) Any of the properties (i)-(iv) is a similarity invariant.

(ii) Any two nonzero invariant linear manifolds of a 1-regular algebra have a nonzero intersection. In particular, a 1-regular algebra is irreducible.

(iii) The weak closure of the restriction of an algebra with any of the properties (i)-(iv) to an invariant subspace has the same property.

(iv) If $A$ is 1-regular then the commutant of $A=\{\lambda \cdot I: \lambda \in \mathbb{C}\}$.

Proof. (ii) Let $L_{1}, L_{2}$ be nonzero invariant linear manifolds for $A$, and suppose that $L_{1} \cap L_{2}=\{0\}$. Then there exists $x, y \neq 0, x \in L_{1}, y \in L_{2}$. We can find $a \in A$ such that

$$
a(x+y)=0, \quad a(x-y) \neq 0 .
$$

The first equation gives $a x+a y=0$, so $a x=0$ and $a y=0$. This contradicts the second equation.

(iv) Let $x \in H$ and let $L_{x}=\{a \in A: a x=0\}$. By 1-regularity $\operatorname{ker} L_{x}=$ $\{\lambda \cdot x: \lambda \in \mathbb{C}\}$. If $c \in A^{\prime}$, the commutant of $A$, and $a \in L_{x}$ then $0=c a x-a c x$, so $c x \in \operatorname{ker} L_{x}$. Thus $c$ leaves every subspace of $H$ invariant and so must be scalar.

Corollary 3. (i) The only commutative 1-regular algebra is the complex numbers.

(ii) The only 1-regular $W^{*}$-algebra is $B(H)$.

Proof. (ii) If $A$ is a 1-regular $W^{*}$-algebra, then $A^{\prime}=\mathbb{C} \cdot I$, so $A=A^{\prime \prime}=B(H)$ by the double commutant theorem.

If $x, y \in H$ then the operator $x \otimes y: H \rightarrow H$ is defined by $x \otimes y(z)=$ $(z, y) x$. Let $K$ be a subspace of $H$. We write $K \otimes K \subseteq A$ if $x \otimes y \in A$ for all $x, y \in K$. This is equivalent to $P_{K} B(H) P_{K} \subseteq A$ where $P_{K}$ is the projection onto $K$.

Theorem 4. Let $A$ be $F$-regular and let $K$ be an atom of $\operatorname{Lat}(A)$, the lattice of invariant subspaces of $A$. Then $K \otimes K \subseteq A$.

Proof. Let $x, y \in K$ and let $y^{\perp}$ be the orthogonal complement of $y$. For each finite-dimensional subspace $M$ of $y^{\perp}$ there exists $a \in A$ such that $a M=\{0\}$ and $a y \neq\{0\}$. Consider $\overline{\mathrm{sp}}\{b a y: b \in A\}$. This is an invariant subspace of $A$ contained in $K$, so it equals $K$. Thus we may choose $a_{M} \in A$ such that $a_{M} M=\{0\}$ and $\left\|a_{M} y-x\right\|<1 /(\operatorname{dim} M)$. Consider the net $\left\{a_{M}\right\}$. On $y^{\perp}$ it converges strongly to 0 and $a_{M} y \stackrel{\|\|}{\rightarrow} x$. Therefore, $a_{M} \stackrel{\text { strongly }}{\rightarrow} x \otimes y /\|y\|^{2}$. Since $A$ is strongly closed, $x \otimes y \in A$. 
Note that $A$ is regular iff for all $y$ there is an $x \neq 0$ such that $x \otimes y \in A$. Recall that if $\mathscr{L}$ is a lattice of projections then $\operatorname{Alg}(\mathscr{L})$ is the algebra of all operators which leave the subspaces in $\mathscr{L}$ invariant. Also if $\left\{P_{i}\right\}$ is a family of projections then $\bigvee P_{i}$ and $\bigwedge P_{i}$ denote their l.u.b. and g.l.b., respectively.

Lemma 5. Let $\mathcal{N}$ be a complete nest of subspaces of $H$, and suppose that $\operatorname{Alg}(\mathcal{N})$ is regular. Then $\mathscr{N}$ contains a minimal element.

Proof. By [3, Proposition 2.12] we can find $y \in H$ such that $P y \neq 0$ for all $P \in \mathscr{N}, P \neq 0$. There exists $x \neq 0$ such that $x \otimes y \in A$. For each $P \in \mathscr{N}$ we must have $(1-P) x \otimes P y=0$ since $x \otimes y$ leaves $P$ invariant. Since $P y \neq 0$ for $P \neq 0$, this implies that $(1-P) x=0$ for $P \neq 0$ or $P x=x$. Thus $x \in \bigwedge_{P \in \mathcal{N}, P \neq 0} P$ which is the minimal element of $\mathscr{N}$.

Lemma 6. If $A$ is a regular algebra then $\operatorname{Lat}(A)$ contains an atom.

Proof. By Zorn's lemma we may pick a maximal chain $\mathscr{C}$ in $\operatorname{Lat}(A)$. Then $A \subseteq \mathrm{Alg}(\mathscr{C})$ and so $\mathscr{C}$ has a minimal element. By maximality of $\mathscr{C}$ this minimal element is an atom of $\operatorname{Lat}(A)$.

Theorem 7. If $A$ is an IC-regular algebra then Lat $(A)$ contains a minimal element.

Proof. If $A$ has no (proper) invariant subspace, the result is immediate. Suppose $A$ has a nonzero invariant subspace $K$ of infinite codimension. Pick $y \in$ $K$, and let $y^{\perp}$ be the complement of $y$ in $K$. Since $y^{\perp}$ has infinite codimension in $H$, we can find $a \in A$ such that $a y^{\perp}=\{0\}$ and $x=a y \neq 0$. Consider $A_{K}$, the weak closure of the restriction of $A$ to $K$. Then $a$ restricted to $K$ is $x \otimes y /\|y\|^{2} \in A_{K}$. Thus $A_{K}$ is regular. We have $\operatorname{Lat}\left(A_{K}\right)=[0, K] \subseteq \operatorname{Lat}(A)$. By Lemma 6, Lat $\left(A_{K}\right)$ contains an atom. This must be an atom in $\operatorname{Lat}(A)$.

We will now exclude the case that every nonzero invariant subspace of $A$ has finite codimension. In that case every invariant subspace of $A^{*}$ is finite dimensional. We can find a sequence $\left\{P_{n}\right\} \subseteq \operatorname{Lat}\left(A^{*}\right)$ such that $\operatorname{dim} P_{n}<\infty$ and $\bigvee P_{n}=I$. Then $A^{*}$ is contained in a triangular algebra of block matrices

$$
\left[\begin{array}{cccc}
A_{11} & A_{12} & \cdot & \cdots \\
0 & A_{22} & \cdot & \cdots \\
0 & 0 & A_{33} & \cdots \\
\cdot & \cdot & \cdot & \cdots \\
\cdot & \cdot & \cdot & \cdots
\end{array}\right]
$$

where the dimensions of each block are finite. So $A$ is contained in a lower triangular algebra of block matrices

$$
\left[\begin{array}{cccc}
B_{11} & 0 & \cdot & \cdots \\
B_{21} & B_{22} & 0 & \cdots \\
B_{31} & B_{32} & B_{33} & \cdots \\
\cdot & \cdot & \cdot & \cdots \\
\cdot & \cdot & \cdot & \cdots
\end{array}\right]
$$

with respect to some orthonormal basis $e_{1}, e_{2}, e_{3}, \ldots$ of $H$. This allows us to conclude that $A$ cannot be IC-regular as follows. Choose an infinitedimensional subspace $M$ of $H$ which does not contain any nonzero vectors of the form $c_{1} e_{1}+\cdots+c_{m} e_{m}$. Then no nonzero $a \in A$ can annihilate $M^{\perp}$. 
Thus $\operatorname{Lat}(A)$ contains an atom. Now suppose that $M$ is any invariant subspace of $A$. Then $A_{M}$ is IC-regular or regular in case $M$ is finite dimensional. Thus $M$ contains an atom of $\operatorname{Lat}(A)$.

Let $P, Q$ be atoms of $\operatorname{Lat}(A)$. Then $P \in A$ by Theorem 4 , and since $Q \in \operatorname{Lat}(A), Q$ is an invariant subspace for the selfadjoint operator $P$ and so $Q$ reduces $P$. Therefore $P, Q$ commute. But $P \wedge Q=0$, so $P \perp Q$. Now $A_{P \oplus Q}$ is IC-regular or regular and reducible. This is a contradiction. Thus there is a unique atom and every invariant subspace contains this atom.

Let $e_{1}, e_{2}, \ldots$ be an O.N.B. for $H$ and let $P_{k}$ be the projection onto $\operatorname{sp}\left\{e_{1}, e_{2}, \ldots, e_{k}\right\}$. We have that $P_{k} \stackrel{s}{\rightarrow} I$. Recall that the Grammian of $n$ vectors $x_{1}, \ldots, x_{n}$ is the determinant of the matrix $\left[\left(x_{i}, x_{j}\right)\right]$. The vectors $x_{1}, x_{2}, \ldots, x_{n}$ are linearly independent iff $G \neq 0$.

Lemma 8. Suppose that $x_{1}, x_{2}, \ldots, x_{n}$ are linearly independent vectors in $H$. Then there is a $k$ such that $P_{k} x_{1}, P_{k} x_{2}, \ldots, P_{k} x_{n}$ are linearly independent.

Proof. The Grammian is a continuous function.

We can now give an example of an $F$-regular algebra which is not IC-regular.

Example 1. The algebra $A$ of all bounded lower triangular matrices

$$
\left[\begin{array}{cccc}
a_{11} & 0 & \cdot & \cdots \\
a_{21} & a_{22} & \cdot & \cdots \\
a_{31} & a_{32} & a_{33} & \cdots \\
\cdot & \cdot & \cdot & \ldots \\
\cdot & \cdot & \cdot & \ldots
\end{array}\right]
$$

is not IC-regular since $\operatorname{Lat}(A)=\left\{P_{k}^{\perp}: k=0,1,2, \ldots\right\}$ which has no minimal element.

Let $x_{1}, x_{2}, \ldots, x_{n}$ be linearly independent vectors in $H$. There is a $k$ such that $P_{k} x_{1}, \ldots, P_{k} x_{n}$ are linearly independent. Therefore, we can find numbers $a_{k 1}, a_{k 2}, \ldots, a_{k k}$ such that $P_{k} x_{1}, \ldots, P_{k} x_{n-1} \perp\left(a_{k 1}, a_{k 2}, \ldots, a_{k k}, 0, \ldots\right)$ and $P_{k} x_{n}$ is not. Let $a$ be the lower triangular matrix whose only nonzero row is the $k$ th row which is $\left(\bar{a}_{k 1}, \bar{a}_{k 2}, \ldots, \bar{a}_{k k}, 0, \ldots\right)$. Then $a x_{1}=a x_{2}=\cdots=$ $a x_{n-1}=0$ and $a x_{n} \neq 0$.

Theorem 9. Every IC-regular algebra is regular.

Proof. Lat $(A)$ contains a minimal element $K$. Let $J=\{y \in H: x \otimes y \in$ $A, \forall x \in K\}$. We have that $K \subseteq J$ by Theorem 4. Now we have an orthogonal decomposition of the Hilbert space and a corresponding block decomposition for any $a \in A$

$$
a=\left[\begin{array}{ccc}
K & J \theta K & J^{\perp} \\
0 & a_{2} & a_{3} \\
0 & a_{4} & a_{5} \\
0 & a_{6} & a_{7}
\end{array}\right] .
$$

For any $y \in J, x \in K$, and $b \in A,(x \otimes y) b=x \otimes b^{*} y \in A$. So $J$ is an invariant subspace for $A^{*}$. Since

$$
\begin{gathered}
K \\
a^{*}=\left[\begin{array}{ccc}
a_{1}^{*} & 0 & 0 \\
a_{2}^{*} & a_{4}^{*} & a_{6}^{*} \\
a_{3}^{*} & a_{5}^{*} & a_{7}^{*}
\end{array}\right],
\end{gathered}
$$


it follows that $a_{3}^{*}=a_{5}^{*}=0$. So

$$
a=\left[\begin{array}{ccc}
a_{1} & a_{2} & 0 \\
0 & a_{4} & 0 \\
0 & a_{6} & a_{7}
\end{array}\right]
$$

Therefore, $J^{\perp} \in \operatorname{Lat}(A)$, which contradicts the minimality of $K$ unless $J^{\perp}=$ 0 . Thus $J=H$ and $K \otimes H \subseteq A$. It follows that $A$ is regular.

Note that we have proved the following for regular algebras. If $K$ is the minimal element of $\operatorname{Lat}(A)$ then $K \otimes H \subseteq A$.

Corollary 10. A reflexive algebra is IC-regular iff Lat $(A)$ contains a minimal element.

Proof. One way is clear. Suppose $\operatorname{Lat}(A)$ contains a minimal element $Q$. Choose $x \in Q$. Then we verify that, for any $y \in H, x \otimes y \in A$. Let $P \in$ Lat $(A)$. We have $P^{\perp}(x \otimes y) P=P^{\perp} x \otimes P y=0 \otimes P y$ since $Q \subseteq P$. Hence Lat $(A) \subseteq \operatorname{Lat}(x \otimes y)$, so $x \otimes y \in A$ by reflexivity.

Example 2. There are few classes of operator algebras on $H$ which are well understood. The reflexive algebras with a commutative subspace lattice form a broad class of algebras for which considerable information has been obtained [3]. Among these are the nest algebras:

$$
\begin{array}{ll}
\operatorname{Alg}(Z) & \text { Standard } Z \text {-nest, } \\
\operatorname{Alg}(R) & \text { Standard } R \text {-nest, } \\
\operatorname{Alg}(V) & \text { Volterra nest. }
\end{array}
$$

An argument similar to that in the previous example shows each of these algebras is $F$-regular but not IC-regular. The algebra of upper triangular matrices is $\operatorname{Alg}(N)$, which is regular.

Problem. Let $\mathscr{L}$ be a commutative subspace lattice. When is $\operatorname{Alg}(\mathscr{L}) 1$ regular?

The following is our main result concerning 1-regular algebras.

Theorem 11. If $A$ is 1-regular then $A$ is F-regular.

Proof. Assume inductively that if $n$ linearly independent vectors $x_{1}$, $x_{2}, \ldots, x_{n}$ are given then there is an $a \in A$ such that $a x_{1}=\cdots=a x_{n-1}=0$ and $a x_{n} \neq 0$. We will show that given $n+1$ linearly independent vectors $x_{1}, x_{2}, \ldots, x_{n+1}$ there is $a \in A$ such that $a x_{1}=\cdots=a x_{n}=0$ and $a x_{n+1} \neq 0$.

Let $L=\left\{a \in A: a x_{1}=a x_{2}=\cdots=a x_{n-1}=0\right\}$. Then $L$ is a left ideal. If there exists $a \in L$ such that $a x_{n}$ and $a x_{n+1}$ are linearly independent then choose $b \in A$ such that $b a x_{n}=0$ and $b a x_{n+1} \neq 0$ using 1-regularity. Thus we may assume that for every $a \in L$ the vectors $a x_{n}$ and $a x_{n+1}$ are linearly dependent. Now if, for some $a \in L, a x_{n}=0$ and $a x_{n+1} \neq 0$ then we are done, so we assume that for all $a \in A$ there is a $\lambda$ such that $\lambda a x_{n}=a x_{n+1}$.

We will now show that there is a fixed $\lambda$ such that, for all $a \in L, a x_{n+1}=$ $\lambda a x_{n}$ whenever $a x_{n} \neq 0$. Suppose not. Then there exist $\alpha \neq \beta$ such that

$$
\alpha a x_{n}=a x_{n+1}, \quad \beta b x_{n}=b x_{n+1} .
$$

If $a x_{n}$ and $b x_{n}$ are linearly independent then from $a+b \in L$ and $\lambda(a+b) x_{n}=$ $(a+b) x_{n+1}$ we obtain $\lambda a x_{n}+\lambda b x_{n}=\alpha a x_{n}+\beta b x_{n}$, so $\lambda=\alpha=\beta$, which is 
a contradiction. Therefore we may assume that $a x_{n}=\gamma b x_{n}$ for some scalar $\gamma$. Now $a-\gamma b \in L,(a-\gamma b) x_{n}=0$, and $(a-\gamma b) x_{n+1}=\alpha a x_{n}-\gamma \beta b x_{n}=$ $(\alpha-\beta) a x_{n} \neq 0$ since we have assumed that $a x_{n} \neq 0$. Thus $a-\gamma b$ satisfies a previously excluded case in the proof.

Therefore there is a $\lambda$ such that, for all $a \in L, a x_{n+1}-\lambda a x_{n}=0$, so $a\left(x_{n+1}-\lambda x_{n}\right)=0$. Since $x_{n+1}-\lambda x_{n} \notin \operatorname{sp}\left\{x_{1}, x_{2}, \ldots, x_{n-1}\right\}$, this contradicts the inductive hypothesis.

Theorem 12. The only transitive 1-regular algebra is $B(H)$.

Proof. By Theorem 11 the algebra $A$ is $F$-regular and Lat $(A)$ contains the atom $H$. Therefore, $H \otimes H \subseteq A$ by Theorem 4 . Since $A$ is strongly closed, this implies that $A=B(H)$.

Theorem 11 holds for general algebras (real or complex). We now show how to deduce some well-known results. Recall that a subalgebra $A$ of $B(H)$ is called $n$-fold strictly transitive if for each set $\left\{x_{i}\right\}_{i=1}^{n}$ of linearly independent vectors and each set $\left\{y_{i}\right\}_{i=1}^{n}$ of vectors in $H$ there exists $a \in A$ such that $a x_{i}=y_{i}$ for $i=1,2, \ldots, n$.

We can deduce the following important result [9, Theorem 8.2].

Corollary 13. If $A$ is a 2-fold strictly transitive subalgebra of $B(H)$ then $A$ is $n$-fold strictly transitive for all $n$.

Proof. $A$ is clearly 1-regular. Thus $A$ is $F$-regular. We can find $a_{i} \in A$ such that $a_{i} x_{i} \neq 0$ and $a_{i} x_{j}=0$ for $j \neq i$. Then we find $b_{i} \in A$ such that $b_{i} a_{i} x_{i}=y_{i}$. It follows that $\sum_{i=1}^{n} b_{i} a_{i}$ has the required property.

Now suppose that $A$ is weakly closed and strictly transitive. We then follow the proofs of [9, Lemma 8.3 and Theorem 8.4] to conclude that $A$ is 2-fold strictly transitive. Therefore, $A$ is 1-regular, so $A=B(H)$ by Theorem 12 (instead of [9, Theorem 8.2j). This is the Rickart-Yood density theorem.

Corollary 14 [9, Theorem 8.4]. If $A$ is a uniformly closed strictly transitive subalgebra of $B(H)$ then $A$ is strictly dense.

In view of previous results it is of interest to ask whether a 1-regular algebra need contain any nonzero compact operators. Interestingly enough, the answer is negative.

Example 3. We will construct a reflexive operator algebra with a commutative subspace lattice which is 1-regular but which contains no nonzero compact operators. To effect the construction we make use of Arveson's theory $[1,3]$. This example is related to the "thin set" phenomena in classical harmonic analysis $[5,6]$.

Define a sequence $P_{1} \supseteq P_{2} \supseteq P_{3} \supseteq \cdots$ of closed partial orders on the unit interval $[0,1]$ as in Figure 1 .

Thus $P_{n+1}$ is obtained from $P_{n}$ by removing the lower left fourth from each of the squares in $P_{n}$. Set $P=\bigcap_{n=1}^{\infty} P_{n}$. Then $P$ is the graph of a closed partial order on $[0,1]$. Let $\operatorname{Alg}(P)$ be the weakly closed algebra of all operators $T: L^{2}[0,1] \rightarrow L^{2}[0,1]$ which are supported in $P[3$, p. 343]. We then have that $\bigcap_{n=1}^{\infty} A \lg \left(P_{n}\right)=\operatorname{Alg}(P)$.

Associated with each step of the construction is a dyadic decomposition of $[0,1]$ and a natural decomposition of any $T \in B\left(L^{2}[0,1]\right)$ as a $2^{n} \times 2^{n}$ block 


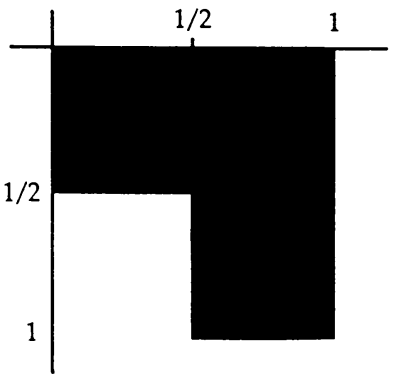

Graph of $P_{1}$

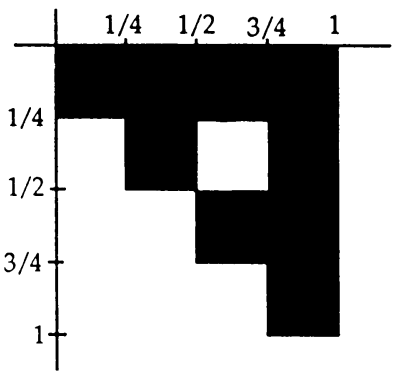

Graph of $P_{2}$

FIGURE 1

matrix $\left[t_{i j}\right]$. Thus $T \in \operatorname{Alg}\left(P_{n}\right)$ iff $t_{i j}=0$ whenever the $i j$ th square has been removed from $P_{n}$. Notice that if a certain square has not been removed from $P_{n}$ then its diagonal is contained in $P$. It is well known that if $f \in L^{\infty}[0,1]$ then the support of $M_{f}$, multiplication by $f$ on $L^{2}[0,1]$, is contained in the diagonal of $[0,1] \times[0,1]$. So we can construct operators with support in $P$ (which interestingly has product measure $=0$ ) by taking $T \in \operatorname{Alg}\left(P_{n}\right)$ such that each $t_{i j}$ is a multiplication operator. Note also that $L^{\infty}[0,1] \subseteq \operatorname{Alg}(P)$.

If

$$
\left[0, \frac{1}{2^{n}}\right],\left[\frac{1}{2^{n}}, \frac{2}{2^{n}}\right], \ldots,\left[\frac{2^{n}-1}{2^{n}}, \frac{1}{2^{n}}\right]
$$

is a dyadic decomposition of $[0,1]$ and $f$ is any function on $[0,1]$, we let $f_{k}:\left[0, \mathrm{i} / 2^{n}\right] \rightarrow \mathbb{C}, k=1,2, \ldots, 2^{n}$, be defined by $f_{k}(x)=f\left((k-1) / 2^{n}+x\right)$.

Lemma 15. Let $f, g$ be linearly independent in $L^{2}[0,1]$. Then there is a dyadic decomposition of $[0,1]$ and functions $\varphi_{1}, \varphi_{2}, \ldots, \varphi_{2^{n}} \in L^{\infty}\left[0,1 / 2^{n}\right]$ such that

$$
\sum_{k=1}^{2^{n}} \varphi_{k} f_{k}=0, \quad \sum_{k=1}^{2^{n}} \varphi_{k} g_{k} \neq 0 .
$$

Proof. Since $L^{\infty}[0,1] \subset \operatorname{Alg}(P)$, we can reduce to the case where $\varepsilon \leq|f|$, $|g| \leq M$ for some constants $\varepsilon, M$, and $\{f \neq 0\}=\{g \neq 0\}$.

Suppose that for every dyadic decomposition of $[0,1]$ the vector

$$
\left[\begin{array}{c}
f_{1}(x) \\
f_{2}(x) \\
\vdots \\
f_{2^{n}}(x)
\end{array}\right]=\alpha_{n}(x)\left[\begin{array}{c}
g_{1}(x) \\
g_{2}(x) \\
\vdots \\
g_{2^{n}}(x)
\end{array}\right]
$$

for some $\alpha_{n}:\left[0,1 / 2^{n}\right] \rightarrow \mathbb{C}$. We may assume that $\alpha_{n}(x)=0$ for $x \notin$ $\bigcup_{k=1}^{2^{n}} \operatorname{supp}\left(f_{k} / g_{k}\right)$. Then we have that $\left\|\alpha_{n}\right\|_{\infty} \leq M / \varepsilon$.

It follows that $f(x)=\lambda_{n}(x) g(x)$ for some periodic $\lambda_{n} \in L^{\infty}[0,1]$ with period $1 / 2^{n}$ and satisfying $\lambda_{n}=\alpha_{n}(x)$ for $x \in\left[0,1 / 2^{n}\right]$. Now $\left\|\lambda_{n}\right\|_{\infty} \leq M / \varepsilon$ in $L^{\infty}$, so we may extract a subsequence $\left\{\lambda_{n_{k}}\right\}$ which converges to some $\lambda$ in the $w^{*}$-topology that $L^{\infty}$ obtains as the dual of $L^{1}$. It is clear that $\lambda$ must be orthogonal to the nonconstant functions of the Haar system [4, p. 27] and thus 
must be constant. We have that $\lambda_{n_{k}} g \stackrel{w^{*}}{\rightarrow} \lambda g$. But since $f=\lambda_{n_{k}} g$, we conclude that $f=\lambda g$ for some constant $\lambda$. This is a contradiction.

Thus there is a dyadic decomposition of $[0,1]$ such that the vectors

$$
\left[\begin{array}{c}
f_{1} \\
f_{2} \\
\vdots \\
f_{2^{n}}
\end{array}\right], \quad\left[\begin{array}{c}
g_{1} \\
g_{2} \\
\vdots \\
g_{2^{n}}
\end{array}\right]
$$

are linearly independent on a subset of $\left[0,1 / 2^{n}\right]$ having positive measure. Let

$$
\varphi_{k}(x)=\overline{g_{k}(x)}-\left(\frac{\sum_{i=1}^{2^{n}} f_{i}(x) \overline{g_{i}(x)}}{\sum_{i=1}^{2^{n}}\left|f_{i}(x)\right|^{2}}\right) \overline{f_{k}(x)}
$$

where $\varphi_{k}$ is taken to be 0 if the denominator is 0 . We see that $\varphi_{k}$ is in $L^{\infty}$ and that $\sum_{k=1}^{2^{n}} \varphi_{k}(x) f_{k}(x)=0$. Suppose now that $\sum_{k=1}^{2^{n}} \varphi_{k}(x) g_{k}(x)=0$ a.e. Then

so

$$
\sum_{k=1}^{2^{n}}\left|g_{k}(x)\right|^{2}-\frac{\left|\sum_{k=1}^{2^{n}} f_{k}(x) \overline{g_{k}(x)}\right|^{2}}{\sum_{k=1}^{2^{n}}\left|f_{k}(x)\right|^{2}}=0
$$

$$
\left(\sum_{k=1}^{2^{n}}\left|g_{k}(x)\right|^{2}\right)\left(\sum_{k=1}^{2^{n}}\left|f_{k}(x)\right|^{2}\right)=\left|\sum_{k=1}^{2^{n}} f_{k}(x) \overline{g_{k}(x)}\right|^{2}
$$

Therefore, we have equality in the Cauchy-Schwarz inequality a.e. It follows that

$$
\left[\begin{array}{c}
f_{1} \\
f_{2} \\
\vdots \\
f_{2^{n}}
\end{array}\right]=\alpha\left[\begin{array}{c}
g_{1} \\
g_{2} \\
\vdots \\
g_{2^{n}}
\end{array}\right],
$$

which is a contradiction. Thus $\sum_{k=1}^{2^{n}} \varphi_{k}(x) g_{k}(x) \neq 0$.

To prove that $\operatorname{Alg}(P)$ is 1-regular let $f, g \in L^{2}[0,1]$ be linearly independent. Choose a dyadic decomposition and functions $\varphi_{1}, \varphi_{2}, \ldots, \varphi_{2^{n}}$ as above such that

$$
\sum_{k=1}^{2^{n}} \varphi_{k} f_{k}=0, \quad \sum_{k=1}^{2^{n}} \varphi_{k} g_{k} \neq 0
$$

Define a $2^{n} \times 2^{n}$ block matrix $\left[t_{i j}\right]$ by $t_{1 j}=M_{\varphi_{j}}$ and $t_{i j}=0$ for $i \neq 1$. Then $T=\left[t_{i j}\right] \in \operatorname{Alg}(P)$ and $T f=0, T g \neq 0$.

Finally we show that $\operatorname{Alg}(P)$ does not contain any nonzero compact operators. Let

$$
K_{n}=\bigcup_{\substack{1 \leq k \leq 2^{n} \\ k \text { odd }}}\left[\frac{k-1}{2^{n}}, \frac{k}{2^{n}}\right] \subset[0,1] .
$$

Let $P_{n}: L^{2}[0,1] \rightarrow L^{2}\left(K_{n}\right)$ be the standard projection. Then $P_{n} \in \operatorname{Lat}\left(\operatorname{Alg}\left(P_{n}\right)\right)$, so $P_{n} \in \operatorname{Lat}(\operatorname{Alg}(P))$. Therefore, $\operatorname{Lat}(\operatorname{Alg}(P))$ contains a sequence $\left\{P_{n}\right\}$ of commuting projections such that, for each subsequence $\left\{P_{n_{i}}\right\}, \bigvee P_{n_{i}}=I$ and $\bigwedge P_{n_{i}}=0$. It follows that $\operatorname{Alg}(P) \cap \mathscr{K}=\{0\}$ [5, Theorem 1.1.4]. 
Problem. If a 1-regular algebra contains a nonzero compact operator must it contain a nonzero finite rank operator?

\section{ACKNOWLEDGMENTS}

We would like to thank the Mathematics Department of Illinois State University for their hospitality and support during the production of this manuscript and Michael Marsalli for a helpful reading and discussion of the manuscript.

\section{REFERENCES}

1. W. Arveson, Operator algebras and invariant subspaces, Ann. of Math. (2) 100 (1974), 433-532.

2. B. Blackadar, $K$-theory for operator algebras, Math. Sci. Res. Inst. Publ., vol. 5, SpringerVerlag, New York, 1986.

3. K. Davidson, Nest algebras, Pitman Res. Notes Math. Ser., vol. 191, Longman Sci. Tech., Harlow, 1988.

4. H. Dym and H. P. McKean, Fourier series and integrals, Academic Press, New York and London, 1972.

5. J. Froelich, Compact operators, invariant subspaces and spectral synthesis, J. Funct. Anal. 81 (1988), 1-37.

6. C. C. Graham and O. C. McGehee, Essays in commutative harmonic analysis, SpringerVerlag, New York, 1979.

7. Y. Katznelson, An introduction to harmonic analysis, Wiley, 1968; reprinted by Dover .

8. S. Power, Analysis in nest algebras, Surveys of recent results in operator theory (J. Conway and B. Morrel, eds.), Pitman Res. Notes Math. Ser., vol. 192, Longman Sci. Tech., Harlow, 1988.

9. H. Radjavi and P. Rosenthal, Invariant subspaces, Ergeb. Math. Grenzgeb. (3), vol. 77, Springer, Berlin and New York, 1973.

Department of Mathematics, University of Houston, Houston, TeXas 77204 